\title{
Inappropriate Sinus Tachycardia by ECG
}

\section{Finding}

National Cancer Institute

\section{Source}

National Cancer Institute. Inappropriate Sinus Tachycardia by ECG Finding. NCI

Thesaurus. Code C62253.

An electrocardiographic finding of supraventricular tachycardia involving the sinus node in which a patient has an atypically high heart rate which cannot be explained by factors such as fever, exercise, hypovolemia, etc. 